\title{
ELLIPTIC GRADIENT ESTIMATES FOR A NONLINEAR HEAT EQUATION AND APPLICATIONS
}

\author{
JIA-YONG WU
}

\begin{abstract}
In this paper, we study elliptic gradient estimates for a nonlinear $f$-heat equation, which is related to the gradient Ricci soliton and the weighted log-Sobolev constant of smooth metric measure spaces. Precisely, we obtain Hamilton's and Souplet-Zhang's gradient estimates for positive solutions to the nonlinear $f$-heat equation only assuming the Bakry-Émery Ricci tensor is bounded below. As applications, we prove parabolic Liouville properties for some kind of ancient solutions to the nonlinear $f$-heat equation. Some special cases are also discussed.
\end{abstract}

\section{INTRODUCTION}

1.1. Background. This is a sequel to our previous work [26]. In that paper we proved elliptic gradient estimates for positive solutions to the $f$-heat equation on smooth metric measure spaces with only the Bakry-Émery Ricci tensor bounded below. We also applied the results to get parabolic Liouville theorems for some ancient solutions to the $f$-heat equation. In this paper we will investigate elliptic gradient estimates and Liouville properties for positive solutions to a nonlinear $f$-heat equation (see equation (1.3) below) on complete smooth metric measure spaces.

Recall that an $n$-dimensional smooth metric measure space $\left(M^{n}, g, e^{-f} d v\right)$ is a complete Riemannian manifold $\left(M^{n}, g\right)$ endowed with a weighted measure $e^{-f} d v$ for some $f \in C^{\infty}(M)$, where $d v$ is the volume element of the metric $g$. The associated $m$-Bakry-Émery Ricci tensor [2] is defined by

$$
R i c_{f}^{m}:=R i c+\nabla^{2} f-\frac{1}{m} d f \otimes d f
$$

for some constant $m>0$, where Ric and $\nabla^{2}$ denote the Ricci tensor and the Hessian of the metric $g$. When $m=\infty$, we have the ( $\infty$-)Bakry-Émery Ricci tensor

$$
R i c_{f}:=R i c+\nabla^{2} f
$$

Date: September 19, 2018.

2010 Mathematics Subject Classification. Primary 53C21, 58J35; Secondary 35B53, $35 \mathrm{~K} 55$.

Key words and phrases. Gradient estimate; Liouville theorem; smooth metric measure space; Bakry-Émery Ricci tensor; log-Sobolev inequality.

This work is partially supported by NSFC (11101267, 11271132). 
The Bochner formula for $\operatorname{Ric}_{f}^{m}$ can be read as (see also [26])

$$
\begin{aligned}
\frac{1}{2} \Delta_{f}|\nabla u|^{2} & =\left|\nabla^{2} u\right|^{2}+\left\langle\nabla \Delta_{f} u, \nabla u\right\rangle+\operatorname{Ric}_{f}(\nabla u, \nabla u) \\
& \geq \frac{\left(\Delta_{f} u\right)^{2}}{m+n}+\left\langle\nabla \Delta_{f} u, \nabla u\right\rangle+\operatorname{Ric}_{f}^{m}(\nabla u, \nabla u)
\end{aligned}
$$

for any $u \in C^{\infty}(M)$. When $m<\infty$, (1.1) could be viewed as the Bochner formula for the Ricci tensor of an $(n+m)$-dimensional manifold. Hence many geometric and topological properties for manifolds with Ricci tensor bounded below can be possibly extended to smooth metric measure spaces with $m$-Bakry-Émery Ricci tensor bounded below, see for example [16, 19]. When $m=\infty$, the $(\infty$-)Bakry-Émery Ricci tensor is related to the gradient Ricci soliton

$$
\operatorname{Ric}_{f}=\lambda g
$$

for some constant $\lambda$, which plays an important role in Hamilton's Ricci flow as it corresponds to the self-similar solution and arises as limits of dilations of singularities in the Ricci flow [11]. A Ricci soliton is said to be shrinking, steady, or expanding according to $\lambda>0, \lambda=0$ or $\lambda<0$. On the gradient estimate, the smooth function $f$ is often called a potential function. We refer [5] and the references therein for further discussions.

On smooth metric measure space $\left(M, g, e^{-f} d v\right)$, the $f$-Laplacian $\Delta_{f}$ is defined by

$$
\Delta_{f}:=\Delta-\nabla f \cdot \nabla,
$$

which is self-adjoint with respect to the weighted measure. The associated $f$-heat equation is defined by

$$
\frac{\partial u}{\partial t}=\Delta_{f} u
$$

If $u$ is independent of time $t$, then it is $f$-harmonic function. In the past few years, various Liouville properties for $f$-harmonic functions were obtained, see for example [3], [16], [17, [21, [23], [25], 27], [28, and the references therein. Recently, the author [26] proved elliptic gradient estimates and parabolic Liouville properties for $f$-heat equation under some assumptions of ( $\infty$-)Bakry-Émery Ricci tensor.

In this paper, we will study analytical and geometrical properties for positive solutions to the equation

$$
\frac{\partial u}{\partial t}=\Delta_{f} u+a u \ln u
$$

where $a \in \mathbb{R}$, on complete smooth metric measure spaces $\left(M, g, e^{-f} d v\right)$ with only the Bakry-Émery Ricci tensor bounded below. Here we assume $M$ has no boundary. It is well-known that all solutions to its Cauchy problem exist for all time. Under the assumption of $R i c_{f}$, we shall prove local elliptic (Hamilton's type and Souplet-Zhang's type) gradient estimates for positive 
solutions to the nonlinear $f$-heat equation (1.3). As applications, we prove parabolic Liouville properties for the nonlinear $f$-heat equation (1.3).

Historically, gradient estimates for the harmonic function on manifolds were discovered by Yau [30] and Cheng-Yau [7] in 1970s. It was extended to the so-called Li-Yau gradient estimate for the heat equation by $\mathrm{Li}$ and $\mathrm{Yau}$ [15] in 1980s. In 1990s, Hamilton [10] gave an elliptic type gradient estimate for the heat equation on closed manifolds, which was later generalized to the non-compact case by Kotschwar [13. In 2006, Souplet and Zhang [22] proved a localized Cheng-Yau type estimate for the heat equation by adding a logarithmic correction term. Integrating Hamilton's or Souplet-Zhang's gradient estimates along space-time paths, their estimates exhibit an interesting phenomenon that one can compare the temperature of two different points at the same time provided the temperature is bounded. However, Li-Yau gradient estimate only provides the comparison at different times.

Equation (1.3) has some relations to the geometrical quantities. On one hand, the time-independent version of (1.3) with constant function $f$ is linked with gradient Ricci solitons, for example, see [20, 29 for detailed explanations. On the other hand, the steady-state version of (1.3) is closely related to weighted log-Sobolev constants of smooth metric measure spaces (Riemmann manifolds case due to Chung-Yau [8]). Recall that, weighted logSobolev constants $S_{M}$, associated to a closed smooth metric measure space $\left(M^{n}, g, e^{-f} d v\right)$, are the smallest positive constants such that the weighted logarithmic-Sobolev inequality

$$
\int_{M} u^{2} \ln \left(u^{2}\right) e^{-f} d v \leq S_{M} \int_{M}|\nabla u|^{2} e^{-f} d v
$$

holds for all smooth function $u$ on $M$ satisfying $\int_{M} u^{2} e^{-f} d v=V_{f}(M)$. In particular, the case of Euclidean space $\mathbb{R}^{n}$ equipped with the Gaussian measure

$$
e^{-f} d v:=(4 \pi)^{-\frac{n}{2}} \exp \left(-\frac{|x|^{2}}{4}\right) d x
$$

is inequivalent to the original log-Sobolev inequality due to L. Gross [9].

If function $u$ achieves the weighted log-Sobolev constant and satisfies $\int_{M} u^{2} e^{-f} d v=V_{f}(M)$, that is,

$$
S_{M}=\frac{\int_{M}|\nabla u|^{2} e^{-f} d v}{\int_{M} u^{2} \ln u^{2} e^{-f} d v}=\inf _{\phi \neq 0} \frac{\int_{M}|\nabla \phi|^{2} e^{-f} d v}{\int_{M} \phi^{2} \ln \phi^{2} e^{-f} d v} .
$$

Using the Lagrange's method with respect to weighted measure $e^{-f} d v$, we have

$$
\frac{-2 \Delta_{f} u}{\int_{M} u^{2} \ln u^{2} e^{-f} d v}-\frac{\int_{M}|\nabla u|^{2} e^{-f} d v}{\left(\int_{M} u^{2} \ln u^{2} e^{-f} d v\right)^{2}}\left(2 u \ln u^{2}+2 u\right)+c_{1} u=0
$$

for some constant $c_{1}$. By the definition of $S_{M}$, this can be reduced to

$$
-\Delta_{f} u-S_{M}\left(u \ln u^{2}+u\right)+c_{2} u=0
$$


for the constant

$$
c_{2}=\frac{c_{1}}{2} \int_{M} u^{2} \ln u^{2} e^{-f} d v .
$$

Notice that multiplying (1.4) by $u$ and integrating it with respect to the weighted measure $e^{-f} d v$, we have

$$
\int_{M}|\nabla u|^{2} e^{-f} d v-S_{M} \int_{M} u^{2}\left(\ln u^{2}+1\right) e^{-f} d v+c_{2} \int_{M} u^{2} e^{-f} d v=0,
$$

which implies $S_{M}=c_{2}$. Therefore (1.4) can be simplified as

$$
\Delta_{f} u+S_{M} u \ln u^{2}=0,
$$

which is an elliptic version of (1.3). For (1.5), if $\operatorname{Ric}_{f}^{m} \geq 0$, using (1.1) instead of the classical Bochner formula for the Ricci tensor and following Chung-Yau's arguments [8], we immediately get

$$
\sup u \leq e^{(n+m) / 2}, \quad|\nabla \ln u|^{2}+S_{M} \ln u^{2} \leq(n+m) S_{M}
$$

and

$$
S_{M} \geq \min \left\{\frac{\lambda_{1}}{8 e}, \frac{1}{(n+m) d^{2}}\right\}
$$

where $\lambda_{1}$ and $d$ denote the first nonzero eigenvalue of the $f$-Laplacian and the diameter of $\left(M^{n}, g, e^{-f} d v\right)$.

1.2. Main results. Our first result gives a local Hamilton's gradient estimate for any positive solution to the equation (1.3).

Theorem 1.1. Let $\left(M, g, e^{-f} d v\right)$ be an $n$-dimensional complete smooth metric measure space. For any point $x_{0} \in M$ and $R \geq 2$, Ric $_{f} \geq-(n-1) K$ for some constant $K \geq 0$ in $B\left(x_{0}, R\right)$. Let $0<u(x, t) \leq D$ for some constant $D$, be a smooth solution to the equation (1.3) in $Q_{R, T} \equiv B\left(x_{0}, R\right) \times\left[t_{0}-T, t_{0}\right] \subset$ $M \times(-\infty, \infty)$, where $t_{0} \in \mathbb{R}$ and $T>0$.

(i) If $a \geq 0$, then there exists a constant $c(n)$ such that

$$
\begin{aligned}
& \frac{|\nabla u|}{\sqrt{u}} \leq c(n) \sqrt{D}\left(\frac{1}{R}+\sqrt{\frac{|\alpha|}{R}}+\frac{1}{\sqrt{t-t_{0}+T}}+\sqrt{K}+\sqrt{c_{1}(n, K, a, D)}\right) \\
& \text { in } Q_{R / 2, T} \text { with } t \neq t_{0}-T \text {, where } c_{1}(n, K, a, D)=\max \{2(n-1) K+ \\
& a(2+\ln D), 0\} \text {. }
\end{aligned}
$$

(ii) If $a<0$, further assuming that $\delta \leq u(x, t) \leq D$ for some constant $\delta>0$, then there exists a constant $c(n)$ such that

$$
\begin{aligned}
& \frac{|\nabla u|}{\sqrt{u}} \leq c(n) \sqrt{D}\left(\frac{1}{R}+\sqrt{\frac{|\alpha|}{R}}+\frac{1}{\sqrt{t-t_{0}+T}}+\sqrt{K}+\sqrt{c_{2}(n, K, a, \delta)}\right) \\
& \text { in } Q_{R / 2, T} \text { with } t \neq t_{0}-T \text {, where } c_{2}(n, K, a, \delta)=\max \{2(n-1) K+ \\
& a(2+\ln \delta), 0\} .
\end{aligned}
$$

Here, $\alpha:=\max _{\left\{x \mid d\left(x, x_{0}\right)=1\right\}} \Delta_{f} r(x)$, where $r(x)$ is the distance function to $x$ from base point $x_{0}$. 
Remark 1.2. Cao, Fayyazuddin Ljungberg and Liu [6] proved Li-Yau type gradient estimates for equation (1.3) with constant function $f$; our results belong to the elliptic type. An distinct feature of Theorem 1.1 is that the gradient estimates hold only assuming the $(\infty$-)Bakry-Émery Ricci tensor is bounded below (without any assumption on $f$ ).

We also remark that our proof is a little different from Yau's original proof [30. In Yau's case, the proof is to compute the evolution of quantity $\ln u$, then multiply by a cut-off function and apply the maximum principle. In our case, we compute the evolution of quantity $u^{1 / 3}$ instead of $\ln u$. Moreover, our proof not only applies some arguments of Souplet-Zhang [22], where the maximum principle in a local space-time supported set is discussed, but also uses some proof tricks of Bailesteanua-Cao-Pulemotov [1], Li [14] and Wei-Wylie's comparison theorem [23].

An immediate application of Theorem 1.1 is the parabolic Liouville property for the nonlinear $f$-heat equation. Similar results appeared in [12].

Theorem 1.3. Let $\left(M, g, e^{-f} d v\right)$ be an $n$-dimensional complete smooth metric measure space with Ric $_{f} \geq 0$.

(i) When $a>0$, if $u(x, t)$ is a positive ancient solution to equation (1.3) (that is, a solution defined in all space and negative time) such that $0<u(x, t) \leq e^{-2}$, then $u$ does not exist.

(ii) When $a<0$, let $u(x, t)$ be a positive ancient solution to equation (1.3). If $e^{-2} \leq u(x, t) \leq D$ for some constant $D<1$, then $u$ does not exist; if $e^{-2} \leq u(x, t) \leq D$ for some constant $D \geq 1$, then $u \equiv 1$.

(iii) When $a=0$, if $u(x, t)$ is a positive ancient solution to equation (1.2) such that $u(x, t)=o\left(\left[r^{1 / 2}(x)+|t|^{1 / 4}\right]^{2}\right)$ near infinity, then $u$ is constant.

Theorem 1.3 immediately implies the following result.

Corollary 1.4. Let $\left(M, g, e^{-f} d v\right)$ be an $n$-dimensional closed smooth metric measure space with Ric f $\geq 0$. If positive smooth function $u(x)$ achieves the weighted log-Sobolev constant $S_{M}$ and satisfies

$$
\int_{M} u^{2} e^{-f} d v=V_{f}(M)
$$

then $u(x)>e^{-2}$.

For more interesting special cases and applications of Theorem 1.1, see Section 3 for furthermore discussion.

Our second result gives a Souplet-Zhang's elliptic gradient estimate for positive solutions to the nonlinear $f$-heat equation (1.3). The proof mainly adopts the arguments of Bailesteanua-Cao-Pulemotov [1], Souplet-Zhang [22] and Brighton [3] (see also [26]). 
Theorem 1.5. Let $\left(M, g, e^{-f} d v\right)$ be an $n$-dimensional complete smooth metric measure space. For any point $x_{0} \in M$ and $R \geq 2, R_{i c} \geq-(n-1) K$ for some constant $K \geq 0$ in $B\left(x_{0}, R\right)$. Let $0<u(x, t) \leq D$ for some constant $D$, be a smooth solution to $f$-heat equation (1.3) in $Q_{R, T} \equiv B\left(x_{0}, R\right) \times\left[t_{0}-\right.$ $\left.T, t_{0}\right] \subset M \times(-\infty, \infty)$, where $t_{0} \in \mathbb{R}$ and $T>0$.

(i) If $a \geq 0$, then there exists a constant $c(n)$ such that

$$
\begin{aligned}
& \frac{|\nabla u|}{u} \leq c(n)\left(\sqrt{\frac{1+|\alpha|}{R}}+\frac{1}{\sqrt{t-t_{0}+T}}+\sqrt{K}+\sqrt{a(\kappa+1)}\right)\left(1+\ln \frac{D}{u}\right) \\
& \quad \text { in } Q_{R / 2, T} \text { with } t \neq t_{0}-T \text {, where } \kappa=\max \{|\ln D|, 1\} .
\end{aligned}
$$

(ii) If $a<0$, then there exists a constant $c(n)$ such that

$$
\begin{aligned}
\frac{|\nabla u|}{u} \leq & c(n)\left(\sqrt{\frac{1+|\alpha|}{R}}+\frac{1}{\sqrt{t-t_{0}+T}}+\sqrt{K}+\sqrt{c_{3}(n, a, K)}+\sqrt{-a \kappa}\right)\left(1+\ln \frac{D}{u}\right) \\
& \text { in } Q_{R / 2, T} \text { with } t \neq t_{0}-T . \text { where } c_{3}(n, a, K)=\max \{a+(n-1) K, 0\}, \\
& \text { and } \kappa=\max \{|\ln D|, 1\} .
\end{aligned}
$$

Here, $\alpha:=\max _{\left\{x \mid d\left(x, x_{0}\right)=1\right\}} \Delta_{f} r(x)$, where $r(x)$ is the distance function to $x$ from base point $x_{0}$.

If $a=0$, theorem recovers the result in [26]. We point out that, similar to Theorem 1.1, gradient estimates of Theorem 1.5 also hold provided that only the Bakry-Emery Ricci tensor is bounded below.

Remark 1.6. In [24] the author proved similar estimates when $m$-BakryÉmery Ricci tensor is bounded below. He also remarked that $m$-BakryÉmery Ricci tensor could be replaced by $(\infty-)$ Bakry-Émery Ricci tensor (see Remark 1.3 (ii) in 24]). Professor Xiang-Dong Li pointed out to me that the remark is not accurate because of the lack of global $f$-Laplacian comparison except some special constraint of $f$ is given. However, Theorem 1.5 corrects my previous remark and provides an answer to this question.

The rest of this paper is organized as follows. In Section 2, we will give some auxiliary lemmas and introduce a space-time cut-off function. These results are prepared to prove Theorem 1.1 and Theorem 1.5. In Section 3, we will give completely detail proofs of Theorem 1.1 by the classical Yau's gradient estimate technique. Then we will apply Theorem 1.1 to prove Theorem 1.3 and Corollary 1.4. Meanwhile we will also discuss some special cases of Theorem 1.1. In Section 4, we will adopt the arguments of Theorem 1.1 in [26] to prove Theorem 1.5 .

\section{BASIC LEMMAS}

In this section, we will give some useful lemmas, which are prepared to prove Theorem 1.1 and Theorem 1.5 in the following sections. Consider the 
nonlinear $f$-heat equation

$$
\frac{\partial u}{\partial t}=\Delta_{f} u+a u \ln u,
$$

where $a$ is a real constant, on an $n$-dimensional complete smooth metric measure space $\left(M, g, e^{-f} d v\right)$. For any point $x_{0} \in M$ and any $R>0$, let

$$
0<u(x, t) \leq D
$$

for some constant $D$, be a smooth solution to (2.1) in $Q_{R, T}: \equiv B\left(x_{0}, R\right) \times$ $\left[t_{0}-T, t_{0}\right] \subset M \times(-\infty, \infty)$, where $t_{0} \in \mathbb{R}$ and $T>0$.

Similar to [14, 18], we introduce a new smooth function

$$
h(x, t):=u^{1 / 3}(x, t)
$$

in $Q_{R, T}$. Then

$$
0<h(x, t) \leq D^{1 / 3}
$$

in $Q_{R, T}$. By (2.1), $h(x, t)$ satisfies

$$
\left(\Delta_{f}-\frac{\partial}{\partial t}\right) h+2 h^{-1}|\nabla h|^{2}+a h \ln h=0 .
$$

Using above, we derive the following evolution formula, which is a generalization of Lemma 2.1 in [12].

Lemma 2.1. Let $\left(M, g, e^{-f} d v\right)$ be an $n$-dimensional complete smooth metric measure space. For any point $x_{0} \in M$ and $R>0$, Ric $_{f} \geq-(n-1) K$ for some constant $K \geq 0$ in $B\left(x_{0}, R\right)$. Let $0<u(x, t) \leq D$ for some constant $D$, be a smooth solution to (2.1) in $Q_{R, T}$. Let

$$
\omega(x, t):=h \cdot|\nabla h|^{2},
$$

where $h:=u^{1 / 3}$. For any $(x, t) \in Q_{R, T}$,

(i) if $a \geq 0$, then $\omega$ satisfies

$$
\left(\Delta_{f}-\frac{\partial}{\partial t}\right) \omega \geq-4 h^{-1}\langle\nabla h, \nabla \omega\rangle+4 h^{-3} \omega^{2}-[2(n-1) K+a \ln D+2 a] \omega .
$$

(ii) if $a<0$, further assuming that $0<\delta \leq u(x, t) \leq D$ for some constant $\delta>0$, then $\omega$ satisfies

$$
\left(\Delta_{f}-\frac{\partial}{\partial t}\right) \omega \geq-4 h^{-1}\langle\nabla h, \nabla \omega\rangle+4 h^{-3} \omega^{2}-[2(n-1) K+a \ln \delta+2 a] \omega .
$$

Proof. Following the computation method of [15], let $e_{1}, e_{2}, \ldots, e_{n}$ be a local orthonormal frame field on $M^{n}$. We adopt the notation that subscripts in $i, j$, and $k$, with $1 \leq i, j, k \leq n$, mean covariant differentiations in the $e_{i}, e_{j}$ and $e_{k}$, directions respectively.

Differentiating $\psi$ in the direction of $e_{i}$, we have

$$
\omega_{j}=h_{j} \cdot|\nabla h|^{2}+2 h h_{i} h_{i j}
$$


and once more differentiating $\psi$ in the direction of $e_{i}$,

$$
\Delta \omega=2 h\left|h_{i j}\right|^{2}+2 h h_{i} h_{i j j}+4 h_{i} h_{j} h_{i j}+h_{i}^{2} h_{j j},
$$

where $h_{i}:=\nabla_{i} h$ and $h_{i j j}:=\nabla_{j} \nabla_{j} \nabla_{i} h$, etc. Hence we have

$$
\begin{aligned}
\Delta_{f} \omega & =\Delta \omega-\langle\nabla f, \nabla \omega\rangle \\
& =2 h\left|h_{i j}\right|^{2}+2 h h_{i} h_{i j j}+4 h_{i} h_{j} h_{i j}+h_{i}^{2} h_{j j}-2 h h_{i j} h_{i} f_{j}-h_{i}^{2} h_{j} f_{j} \\
& =2 h\left|h_{i j}\right|^{2}+2 h h_{i}\left(\Delta_{f} h\right)_{i}+2 h R i c_{f}(\nabla h, \nabla h)+4 h_{i} h_{j} h_{i j}+h_{i}^{2} \Delta_{f} h .
\end{aligned}
$$

By (2.2), we also have

$$
\begin{aligned}
\frac{\partial \omega}{\partial t}= & 2 h \nabla_{i} h \cdot \nabla_{i}\left(\Delta_{f} h+2 h^{-1} h_{j}^{2}+a h \ln h\right)+h_{t} h_{j}^{2} \\
= & 2 h \nabla h \nabla \Delta_{f} h+8 h_{i} h_{j} h_{i j}-4 h^{-1} h_{i}^{4}+2 a h(\ln h+1) h_{i}^{2} \\
& +h_{i}^{2} \Delta_{f} h+2 h^{-1}|\nabla h|^{4}+a h \ln h \cdot h_{i}^{2} \\
= & 2 h \nabla h \nabla \Delta_{f} h+8 h_{i} h_{j} h_{i j}-2 h^{-1} h_{i}^{4}+(3 \ln h+2) a h h_{i}^{2}+h_{i}^{2} \Delta_{f} h .
\end{aligned}
$$

Combining the above two equations, we get

$$
\begin{aligned}
\left(\Delta_{f}-\frac{\partial}{\partial t}\right) \omega= & 2 h\left|h_{i j}\right|^{2}+2 h \operatorname{Ric}_{f_{i j}} h_{i} h_{j}-4 h_{i} h_{j} h_{i j} \\
& +2 h^{-1} h_{i}^{4}-(3 \ln h+2) a h h_{i}^{2} .
\end{aligned}
$$

Since $\operatorname{Ric}_{f} \geq-(n-1) K$ for some constant $K \geq 0$, then

$$
\begin{aligned}
\left(\Delta_{f}-\frac{\partial}{\partial t}\right) \omega \geq & 2 h\left|h_{i j}\right|^{2}+4 h_{i} h_{j} h_{i j}+2 h^{-1} h_{i}^{4}-2(n-1) K \omega \\
& -8 h_{i} h_{j} h_{i j}-(3 \ln h+2) a \omega .
\end{aligned}
$$

Using

$$
2 h\left|h_{i j}\right|^{2}+4 h_{i} h_{j} h_{i j}+2 h^{-1} h_{i}^{4} \geq 0,
$$

we further get

$$
\left(\Delta_{f}-\frac{\partial}{\partial t}\right) \omega \geq-8 h_{i} h_{j} h_{i j}-[2(n-1) K+3 a \ln h+2 a] \omega .
$$

Since (2.3) implies

$$
\omega_{j} h_{j}=2 h h_{i} h_{j} h_{i j}+h_{i}^{4}
$$

using this, (2.4) can be written by

$$
\left(\Delta_{f}-\frac{\partial}{\partial t}\right) \omega \geq 4 h^{-3} \omega^{2}-4 h^{-1}\langle\nabla h, \nabla \omega\rangle-[2(n-1) K+3 a \ln h+2 a] \omega .
$$

Finally, we notice that if $a \geq 0$, then $0<h \leq D^{1 / 3}$ and hence

$$
\ln h \leq 1 / 3 \ln D \text {. }
$$

If $a<0$, then $\delta^{1 / 3} \leq h \leq D^{1 / 3}$ and hence

$$
1 / 3 \ln \delta \leq \ln h \leq 1 / 3 \ln D .
$$

The above two cases imply the desired results. 
For equation (2.1), if we introduce another new function

$$
g=\ln u,
$$

then $g$ satisfies

$$
\left(\Delta_{f}-\frac{\partial}{\partial t}\right) g+|\nabla g|^{2}+a g=0 .
$$

Using this, we can get the following lemma, which is also a generalization of previous results in [22, 24, 26].

Lemma 2.2. Let $\left(M, g, e^{-f} d v\right)$ be an $n$-dimensional complete smooth metric measure space. For any point $x_{0} \in M$ and $R>0$, Ric $_{f} \geq-(n-1) K$ for some constant $K \geq 0$ in $B\left(x_{0}, R\right)$. Let $0<u(x, t) \leq D$ be a smooth solution to equation (2.1) in $Q_{R, T}$. Let $g:=\ln u$ and $\mu:=1+\ln D$. Then for all $(x, t) \in Q_{R, T}$, the function

$$
\omega:=|\nabla \ln (\mu-g)|^{2}=\frac{|\nabla g|^{2}}{(\mu-g)^{2}}
$$

satisfies

$$
\begin{aligned}
\left(\Delta_{f}-\frac{\partial}{\partial t}\right) \omega \geq & \frac{2(g-\ln D)}{\mu-g}\langle\nabla g, \nabla \omega\rangle \\
& +2(\mu-g) \omega^{2}-2(a+(n-1) K) \omega-\frac{2 a g}{\mu-g} \omega .
\end{aligned}
$$

Proof. The proof of lemma is almost the same as that of [24]. In fact, in Lemma 2.1 of [24], if we let $\alpha=1+\ln D$, which means $\alpha=\mu$, then we have $\delta=1$. Therefore from (2.4) of [24], we immediately get (2.6).

In the rest of this section, we introduce a smooth cut-off function originated by Li-Yau [15] (see also [1] and [26]). This will also be used in the proof of our theorems.

Lemma 2.3. Fix $t_{0} \in \mathbb{R}$ and $T>0$. For any $\tau \in\left(t_{0}-T, t_{0}\right]$, there exists a smooth function $\bar{\psi}:[0, \infty) \times\left[t_{0}-T, t_{0}\right] \rightarrow \mathbb{R}$ such that:

(1)

$$
0 \leq \bar{\psi}(r, t) \leq 1
$$

in $[0, R] \times\left[t_{0}-T, t_{0}\right]$, and it is supported in a subset of $[0, R] \times\left[t_{0}-\right.$ $\left.T, t_{0}\right]$.

$$
\bar{\psi}(r, t)=1 \quad \text { and } \quad \frac{\partial \bar{\psi}}{\partial r}(r, t)=0
$$

in $[0, R / 2] \times\left[\tau, t_{0}\right]$ and $[0, R / 2] \times\left[t_{0}-T, t_{0}\right]$, respectively . 
(3)

$$
\left|\frac{\partial \bar{\psi}}{\partial t}\right| \leq \frac{C \bar{\psi}^{\frac{1}{2}}}{\tau-\left(t_{0}-T\right)}
$$

in $[0, \infty) \times\left[t_{0}-T, t_{0}\right]$ for some $C>0$, and $\bar{\psi}\left(r, t_{0}-T\right)=0$ for all $r \in[0, \infty)$.

(4)

$$
-\frac{C_{\epsilon} \bar{\psi}^{\epsilon}}{R} \leq \frac{\partial \bar{\psi}}{\partial r} \leq 0 \quad \text { and } \quad\left|\frac{\partial^{2} \bar{\psi}}{\partial r^{2}}\right| \leq \frac{C_{\epsilon} \bar{\psi}^{\epsilon}}{R^{2}}
$$

in $[0, \infty) \times\left[t_{0}-T, t_{0}\right]$ for each $\epsilon \in(0,1)$ with some constant $C_{\epsilon}$ depending on $\epsilon$.

We remind the readers that Lemma 2.3 is a little different from that of [15] and [22]. Here, the cut-off function was previously used by M. Bailesteanua, X. Cao and A. Pulemotov [1].

\section{Proof of Theorem 1.1}

In this section, we will apply Lemmas 2.1 and 2.3, the localization technique of Souplet-Zhang [22], some tricks of Bailesteanua-Cao-Pulemotov [1], Li [14, Brighton [3] and Jiang [12] to prove Theorem 1.1.

Proof of Theorem 1.1. We only prove the case (i) $a \geq 0$. The case (ii) $a<0$ is similar. Pick any number $\tau \in\left(t_{0}-T, t_{0}\right]$ and choose a cutoff function $\bar{\psi}(r, t)$ satisfying propositions of Lemma 2.3. We will show that (1.6) holds at the space-time point $(x, \tau)$ for all $x$ such that $d\left(x, x_{0}\right)<R / 2$, where $R \geq 2$. Since $\tau$ is arbitrary, the conclusion then follows.

Introduce a cutoff function $\psi: M \times\left[t_{0}-T, t_{0}\right] \rightarrow \mathbb{R}$, such that

$$
\psi=\bar{\psi}\left(d\left(x, x_{0}\right), t\right) \equiv \psi(r, t) .
$$

Then, $\psi(x, t)$ is supported in $Q_{R, T}$. Our aim is to estimate $\left(\Delta_{f}-\frac{\partial}{\partial t}\right)(\psi \omega)$ and carefully analyze the result at a space-time point where the function $\psi \omega$ attains its maximum.

By Lemma 2.1(i), we can calculate that

$$
\begin{aligned}
\left(\Delta_{f}-\frac{\partial}{\partial t}\right)(\psi \omega)+ & \left(4 h^{-1} \nabla h-2 \frac{\nabla \psi}{\psi}\right) \cdot \nabla(\psi \omega) \\
\geq & 4 h^{-3} \omega^{2} \psi+4 h^{-1}\langle\nabla h, \nabla \psi\rangle \omega-2 \frac{|\nabla \psi|^{2}}{\psi} \omega \\
& +\left(\Delta_{f} \psi\right) \omega-\psi_{t} \omega-[2(n-1) K+a \ln D+2 a] \psi \omega .
\end{aligned}
$$

Let $\left(x_{1}, t_{1}\right)$ be a maximum space-time point for $\psi \omega$ in the closed set

$$
\left\{(x, t) \in M \times\left[t_{0}-T, \tau\right] \mid d\left(x, x_{0}\right) \leq R\right\} .
$$

Assume that $(\psi \omega)\left(x_{1}, t_{1}\right)>0$; otherwise, $\omega(x, \tau) \leq 0$ and (1.6) naturally holds at $(x, \tau)$ whenever $d\left(x, x_{0}\right)<\frac{R}{2}$. Here $t_{1} \neq t_{0}-T$, since we assume $(\psi \omega)\left(x_{1}, t_{1}\right)>0$. We can also assume that function $\psi(x, t)$ is smooth at 
$\left(x_{1}, t_{1}\right)$ due to the standard Calabi's argument [4]. Since $\left(x_{1}, t_{1}\right)$ is a maximum space-time point, at this point,

$$
\Delta_{f}(\psi \omega) \leq 0, \quad(\psi \omega)_{t} \geq 0 \quad \text { and } \quad \nabla(\psi \omega)=0 .
$$

Using these, (3.1) at space-time $\left(x_{1}, t_{1}\right)$ can be simplified as

$$
\begin{aligned}
4 \omega^{2} \psi \leq & \left(-4 h^{2}\langle\nabla h, \nabla \psi\rangle+2 \frac{|\nabla \psi|^{2}}{\psi} h^{3}\right) \omega-\left(\Delta_{f} \psi\right) h^{3} \omega+\psi_{t} h^{3} \omega \\
& +c_{1}(n, K, a, D) \cdot \psi h^{3} \omega,
\end{aligned}
$$

where $c_{1}(n, K, a, D):=\max \{2(n-1) K+a \ln D+2 a, 0\}$.

We apply (3.2) to prove the theorem. If $x_{1} \in B\left(x_{0}, 1\right)$, then $\psi$ is constant in space direction in $B\left(x_{0}, R / 2\right)$ according to our assumption, where $R \geq 2$. So at $\left(x_{1}, t_{1}\right),(3.2)$ yields

$$
\begin{aligned}
\omega & \leq D\left(\frac{1}{4} \cdot \frac{\psi_{t}}{\psi}+\frac{c_{1}(n, K, a, D)}{4}\right) \\
& \leq D\left(\frac{C}{\tau-\left(t_{0}-T\right)}+\frac{c_{1}(n, K, a, D)}{4}\right),
\end{aligned}
$$

where we used proposition (3) of Lemma 2.3. Since $\psi(x, \tau)=1$ when $d\left(x, x_{0}\right)<R / 2$ by the proposition (2) of Lemma 2.3, the above estimate indeed gives

$$
\begin{aligned}
\omega(x, \tau)=(\psi \omega)(x, \tau) & \leq(\psi \omega)\left(x_{1}, t_{1}\right) \\
& \leq \omega\left(x_{1}, t_{1}\right) \\
& \leq D\left(\frac{C}{\tau-\left(t_{0}-T\right)}+\frac{c_{1}(n, K, a, D)}{4}\right)
\end{aligned}
$$

for all $x \in M$ such that $d\left(x, x_{0}\right)<R / 2$. By the definition of $w(x, \tau)$ and the fact that $\tau \in\left(t_{0}-T, t_{0}\right]$ was chosen arbitrarily, we prove that

$$
\frac{|\nabla u|}{\sqrt{u}}(x, t) \leq \sqrt{D}\left(\frac{C}{\sqrt{t-t_{0}+T}}+\frac{1}{2} \sqrt{c_{1}(n, K, a, D)}\right)
$$

for all $(x, t) \in Q_{R / 2, T}$ with $t \neq t_{0}-T$. This implies (1.6).

Now, we assume $x_{1} \notin B\left(x_{0}, 1\right)$. Since $\operatorname{Ric}_{f} \geq-(n-1) K$ and $r\left(x_{1}, x_{0}\right) \geq 1$ in $B\left(x_{0}, R\right)$, we have the $f$-Laplacian comparison (see Theorem 3.1 in [23])

$$
\Delta_{f} r\left(x_{1}\right) \leq \alpha+(n-1) K(R-1),
$$

where $\alpha:=\max _{\left\{x \mid d\left(x, x_{0}\right)=1\right\}} \Delta_{f} r(x)$. This comparison theorem holds without any grow condition of $f$, which is critical in our latter proof. Below we will estimate upper bounds for each term of the right-hand side of (3.2), similar to the arguments of Souplet-Zhang [22]. Meanwhile, we also repeatedly use the Young's inequality

$$
a_{1} a_{2} \leq \frac{a_{1}^{p}}{p}+\frac{a_{2}^{q}}{q}, \quad \forall a_{1}, a_{2}, p, q>0 \text { with } \frac{1}{p}+\frac{1}{q}=1 .
$$


In the following $c$ denotes a constant depending only on $n$ whose value may change from line to line.

First, we have the estimates of first term of the right hand side of (3.2):

$$
\begin{aligned}
-4 h^{2}\langle\nabla h, \nabla \psi\rangle \omega & \leq 4 h^{3 / 2} \cdot|\nabla \psi| \cdot \omega^{3 / 2} \\
& \leq 4 D^{1 / 2} \cdot|\nabla \psi| \psi^{-3 / 4} \cdot\left(\psi \omega^{2}\right)^{3 / 4} \\
& \leq \frac{3}{5} \psi \omega^{2}+c D^{2} \frac{|\nabla \psi|^{4}}{\psi^{3}} \\
& \leq \frac{3}{5} \psi \omega^{2}+c \frac{D^{2}}{R^{4}}
\end{aligned}
$$

For the second term of the right hand side of (3.2), we have

$$
\begin{aligned}
2 \frac{|\nabla \psi|^{2}}{\psi} h^{3} \omega & \leq 2 D \cdot|\nabla \psi|^{2} \psi^{-3 / 2} \cdot \psi^{1 / 2} \omega \\
& \leq \frac{3}{5} \psi \omega^{2}+c D^{2} \frac{|\nabla \psi|^{4}}{\psi^{3}} \\
& \leq \frac{3}{5} \psi \omega^{2}+c \frac{D^{2}}{R^{4}} .
\end{aligned}
$$

For the third term of the right hand side of (3.2), since $\psi$ is a radial function, then at $\left(x_{1}, t_{1}\right)$, using (3.3) we have

$$
\begin{aligned}
-\left(\Delta_{f} \psi\right) h^{3} \omega & =-\left[\left(\partial_{r} \psi\right) \Delta_{f} r+\left(\partial_{r}^{2} \psi\right) \cdot|\nabla r|^{2}\right] h^{3} \omega \\
& \leq-\left[\partial_{r} \psi(\alpha+(n-1) K(R-1))+\partial_{r}^{2} \psi\right] h^{3} \omega \\
& \leq D\left[\left|\partial_{r}^{2} \psi\right|+(|\alpha|+(n-1) K(R-1))\left|\partial_{r} \psi\right|\right] \omega \\
& =D \psi^{1 / 2} \omega \frac{\left|\partial_{r}^{2} \psi\right|}{\psi^{1 / 2}}+D(|\alpha|+(n-1) K(R-1)) \psi^{1 / 2} \omega \frac{\left|\partial_{r} \psi\right|}{\psi^{1 / 2}} \\
& \leq \frac{3}{5} \psi \omega^{2}+c D^{2}\left[\left(\frac{\left|\partial_{r}^{2} \psi\right|}{\psi^{1 / 2}}\right)^{2}+\left(\frac{|\alpha| \cdot\left|\partial_{r} \psi\right|}{\psi^{1 / 2}}\right)^{2}+\left(\frac{K(R-1)\left|\partial_{r} \psi\right|}{\psi^{1 / 2}}\right)^{2}\right] \\
& \leq \frac{3}{5} \psi \omega^{2}+c \frac{D^{2}}{R^{4}}+c \frac{\alpha^{2} D^{2}}{R^{2}}+c K^{2} D^{2},
\end{aligned}
$$

where in the last inequality we used proposition (4) of Lemma 2.3.

Then we estimate the fourth term of the right hand side of (3.2):

$$
\begin{aligned}
\left|\psi_{t}\right| h^{3} \omega & =\psi^{1 / 2} \omega \frac{h^{3}\left|\psi_{t}\right|}{\psi^{1 / 2}} \\
& \leq \frac{3}{5}\left(\psi^{1 / 2} \omega\right)^{2}+c\left(\frac{h^{3}\left|\psi_{t}\right|}{\psi^{1 / 2}}\right)^{2} \\
& \leq \frac{3}{5} \psi \omega^{2}+\frac{c D^{2}}{\left(\tau-t_{0}+T\right)^{2}} .
\end{aligned}
$$


Finally, we estimate the last term of the right hand side of (3.2):

$$
c_{1}(n, K, a, D) \psi h^{3} \omega \leq \frac{3}{5} \psi \omega^{2}+c D^{2} c_{1}^{2}(n, K, a, D)
$$

We now substitute (3.4)-(3.8) into the right hand side of (3.2), and get that

$$
\psi \omega^{2} \leq c D^{2}\left(\frac{1}{R^{4}}+\frac{\alpha^{2}}{R^{2}}+\frac{1}{\left(\tau-t_{0}+T\right)^{2}}+K^{2}+c_{1}^{2}(n, K, a, D)\right)
$$

at $\left(x_{1}, t_{1}\right)$. This implies that

$$
\begin{aligned}
\left(\psi^{2} \omega^{2}\right)\left(x_{1}, t_{1}\right) & \leq\left(\psi \omega^{2}\right)\left(x_{1}, t_{1}\right) \\
& \leq c D^{2}\left(\frac{1}{R^{4}}+\frac{\alpha^{2}}{R^{2}}+\frac{1}{\left(\tau-t_{0}+T\right)^{2}}+K^{2}+c_{1}^{2}(n, K, a, D)\right) .
\end{aligned}
$$

Since $\psi(x, \tau)=1$ when $d\left(x, x_{0}\right)<R / 2$ by the proposition (2) of Lemma 2.3, from the above estimate, we have

$$
\begin{aligned}
\omega(x, \tau) & =(\psi \omega)(x, \tau) \\
& \leq(\psi \omega)\left(x_{1}, t_{1}\right) \\
& \leq c D\left(\frac{1}{R^{2}}+\frac{|\alpha|}{R}+\frac{1}{\tau-t_{0}+T}+K+c_{1}(n, K, a, D)\right)
\end{aligned}
$$

for all $x \in M$ such that $d\left(x, x_{0}\right)<R / 2$. By the definition of $w(x, \tau)$ and the fact that $\tau \in\left(t_{0}-T, t_{0}\right]$ was chosen arbitrarily, we in fact show that

$$
\sqrt{h}|\nabla h|(x, t) \leq c \sqrt{D}\left(\frac{1}{R}+\frac{\sqrt{|\alpha|}}{\sqrt{R}}+\frac{1}{\sqrt{t-t_{0}+T}}+\sqrt{K}+\sqrt{c_{1}(n, K, a, D)}\right)
$$

for all $(x, t) \in Q_{R / 2, T} \equiv B\left(x_{0}, R / 2\right) \times\left[t_{0}-T, t_{0}\right]$ with $t \neq t_{0}-T$. We have finished the proof of theorem since $h=u^{1 / 3}$ and $R \geq 2$.

In particular, if $a=0$, Theorem 1.1 implies a local elliptic gradient estimate for the $f$-heat equation:

$$
\frac{|\nabla u|}{\sqrt{u}} \leq c(n) \sqrt{D}\left(\frac{1}{R}+\sqrt{\frac{|\alpha|}{R}}+\frac{1}{\sqrt{t-t_{0}+T}}+\sqrt{K}\right)
$$

in $Q_{R / 2, T}$ with $t \neq t_{0}-T$, for any $R \geq 2$. Compared with author's recent result [26], though (3.10]) is not sharp from Example 1.2 of [26], it seems to be a new form of elliptic type gradient estimates for $f$-heat equation.

Furthermore, if $a=0$ and $f$ is constant, by using the classical Laplacian comparison $\Delta r \leq(n-1)(1 / r+\sqrt{K})$ instead of Wei-Wylie's $f$-Laplacian 
comparison (see (3.3)), the proof of Theorem 1.1 in fact implies the following gradient estimate for the heat equation:

$$
\frac{|\nabla u|}{\sqrt{u}} \leq c(n) \sqrt{D}\left(\frac{1}{R}+\frac{1}{\sqrt{t-t_{0}+T}}+\sqrt{K}\right)
$$

in $Q_{R / 2, T}$ with $t \neq t_{0}-T$, for all $R>0$. Compared with Hamilton's estimate [10] and Souplet-Zhang's estimate [22] for the heat equation, this elliptic gradient estimate seems to be new.

Moreover, gradient estimate (3.10) implies

Corollary 3.1. Let $\left(M, g, e^{-f} d v\right)$ be an $n$-dimensional complete smooth metric measure space with Ric $_{f} \geq-(n-1) K$ for some constant $K \geq 0$. If $u(x, t)$ is a positive ancient solution to the $f$-heat equation (1.2) such that $0<u(x, t) \leq D$ for some constant $D$, then $|\nabla u| \leq c(n) D \sqrt{K}$.

Remark 3.2. Corollary 3.1implies Brighton's result [3]: any positive bounded $f$-harmonic function on complete noncompact smooth metric measure spaces with Ric $_{f} \geq 0$ must be constant.

In the rest of this section, we shall apply Theorem 1.1 to prove Theorem 1.3. Corollary 1.4 and Corollary 3.1 .

Proof of Theorem 1.3. When $a>0$, since $K=0$ and $D=e^{-2}$, then $c_{1}(n, K, a, D)=0$. Fixing any space-time point $\left(x_{0}, t_{0}\right)$ and using Theorem 1.1 for $0<u \leq e^{-2}$ in the set $B\left(x_{0}, R\right) \times\left(t_{0}-R^{2}, t_{0}\right]$, we have

$$
\frac{|\nabla u|}{\sqrt{u}}\left(x_{0}, t_{0}\right) \leq c(n) e^{-2}\left(\sqrt{\frac{1+|\alpha|}{R}}+\frac{1}{R}\right)
$$

for all $R \geq 2$. Letting $R \rightarrow \infty$, then

$$
\left|\nabla u\left(x_{0}, t_{0}\right)\right|=0 .
$$

Since $\left(x_{0}, t_{0}\right)$ is arbitrary, $u$ must be constant in $x$. By equation (1.3), then

$$
\frac{d u}{d t}=a u \ln u \text {. }
$$

Solving this ODE, we get

$$
u=\exp \left(c e^{a t}\right)
$$

where $c$ is some constant and $a>0$. For such a solution, if we let $t \rightarrow-\infty$, then

$$
u=\exp \left(c e^{a t}\right) \rightarrow 1,
$$

which is contradiction with the theorem assumption: $0<u(x, t) \leq e^{-2}$. Therefore such $u$ does not exist. 
When $a<0$, since $K=0$ and $\delta=e^{-2}$, then $c_{2}(n, K, a, D)=0$. For any space-time point $\left(x_{0}, t_{0}\right)$, we apply Theorem 1.1 for $e^{-2} \leq u(x, t) \leq D$ in the set $B\left(x_{0}, R\right) \times\left(t_{0}-R^{2}, t_{0}\right]$,

$$
\frac{|\nabla u|}{\sqrt{u}}\left(x_{0}, t_{0}\right) \leq c(n) \sqrt{D}\left(\sqrt{\frac{1+|\alpha|}{R}}+\frac{1}{R}\right)
$$

for all $R \geq 2$. Similar to the above arguments, letting $R \rightarrow \infty$, then $u$ is constant in $x$, and $u=\exp \left(c e^{a t}\right)$ for some constant $c$. When $t \rightarrow-\infty$, we observe that: $u=\exp \left(c e^{a t}\right) \rightarrow+\infty$ if $c>0 ; u=\exp \left(c e^{a t}\right) \rightarrow 0$ if $c<0 ; u=$ 1 if $c=0$. Moreover, the theorem assumption requires $e^{-2} \leq u(x, t) \leq D$. Hence $u$ only exists when $D \geq 1$ and the desired result follows.

When $a=0, K=0$, and assume that $u(x, t)$ is a positive ancient solution to equation (1.2) such that $u(x, t)=o\left(\left[r^{1 / 2}(x)+|t|^{1 / 4}\right]^{2}\right)$ near infinity. Fixing any space-time $\left(x_{0}, t_{0}\right)$ and using (3.10) for $u$ on the set $B\left(x_{0}, R\right) \times$ $\left(t_{0}-R^{2}, t_{0}\right]$, we obtain

$$
\frac{|\nabla u|}{\sqrt{u}}\left(x_{0}, t_{0}\right) \leq c(n)\left(\sqrt{\frac{1+|\alpha|}{R}}+\frac{1}{R}\right) \cdot o(\sqrt{R})
$$

for all $R \geq 2$. Letting $R \rightarrow \infty$, it follows that

$$
\left|\nabla u\left(x_{0}, t_{0}\right)\right|=0 .
$$

Since $\left(x_{0}, t_{0}\right)$ is arbitrary, we get $u$ is constant in space-time.

Theorem 1.3 in fact implies Corollary 1.4 .

Proof of Corollary 1.4. If positive smooth function $u(x)$ achieves the weighted $\log$-Sobolev constant $S_{M}$ and satisfies $\int_{M} u^{2} e^{-f} d v=V_{f}(M)$, then from the introduction above, $u(x)$ satisfies elliptic equation (1.5). Assume that our conclusion is incorrect, that is, $0<u \leq e^{-2}$. Since $S_{M}>0$, by Theorem 1.3 Case (i), there does not exist such function $u$ satisfying (1.5). This is a contradiction.

Proof of Corollary [3.1. When $a=0$, for any space-time point $\left(x_{0}, t_{0}\right)$, using estimate (3.10) for $0<u \leq D$ in the set $B\left(x_{0}, R\right) \times\left(t_{0}-R^{2}, t_{0}\right]$,

$$
\frac{|\nabla u|}{\sqrt{u}}\left(x_{0}, t_{0}\right) \leq c(n) \sqrt{D}\left(\sqrt{\frac{1+|\alpha|}{R}}+\frac{1}{R}+\sqrt{K}\right)
$$

for all $R \geq 2$. Letting $R \rightarrow \infty$, then

$$
\left|\nabla u\left(x_{0}, t_{0}\right)\right| \leq c(n) D \sqrt{K}
$$

Since $\left(x_{0}, t_{0}\right)$ is arbitrary, the result follows. 


\section{Proof of Theorem 1.5}

In this section, we will prove Theorem 1.5. The proof is analogous to Theorem 1.1 in [26]. For the readers convenience, we provide a detailed proof. Compared with the previous proof, here we need to carefully deal with an extra nonlinear term.

Proof of Theorem 1.5. We only consider the case $a \geq 0$. The case $a<0$ is similar. Using Lemma 2.2, we calculate that

$$
\begin{aligned}
& \Delta_{f}(\psi \omega)-\frac{2(g-\ln D)}{\mu-g} \nabla g \cdot \nabla(\psi \omega)-2 \frac{\nabla \psi}{\psi} \cdot \nabla(\psi \omega)-(\psi \omega)_{t} \\
\geq & 2 \psi(\mu-g) \omega^{2}-\left[\frac{2(g-\ln D)}{\mu-g} \nabla g \cdot \nabla \psi\right] \omega-2 \frac{|\nabla \psi|^{2}}{\psi} \omega \\
& +\left(\Delta_{f} \psi\right) \omega-\psi_{t} \omega-2(a+(n-1) K) \psi \omega-\frac{2 a g}{\mu-g} \psi \omega .
\end{aligned}
$$

Let $\left(x_{1}, t_{1}\right)$ be a point where $\psi \omega$ achieves the maximum.

We first consider the case $x_{1} \notin B\left(x_{0}, 1\right)$. By Li-Yau [15], without loss of generality we assume that $x_{1}$ is not in the cut-locus of $M$. Then at this point, we have

$$
\Delta_{f}(\psi \omega) \leq 0, \quad(\psi \omega)_{t} \geq 0, \quad \nabla(\psi \omega)=0
$$

Hence by (4.1), at $\left(x_{1}, t_{1}\right)$, we get

$$
\begin{aligned}
2 \psi(\mu-g) \omega^{2} \leq & \left\{\left(\frac{2(g-\ln D)}{\mu-g} \nabla g \cdot \nabla \psi\right) \omega+2 \frac{|\nabla \psi|^{2}}{\psi} \omega-\left(\Delta_{f} \psi\right) \omega\right. \\
& \left.+\psi_{t} \omega+2(a+(n-1) K) \psi \omega+\frac{2 a g}{\mu-g} \psi \omega\right\} .
\end{aligned}
$$

We will carefully estimate the upper bounds for each term of the right-hand side of (4.2). Similar to arguments of Section 3, we still repeatedly use the Young's inequality. For the first term of right hand side of (4.2), we have

$$
\left(\frac{2(g-\ln D)}{\mu-g} \nabla g \cdot \nabla \psi\right) \omega \leq(\mu-g) \psi \omega^{2}+c \frac{|\ln D-g|}{R^{4}}
$$

For the second term of the right hand side of (4.2), we get

$$
2 \frac{|\nabla \psi|^{2}}{\psi} \omega \leq \frac{1}{10} \psi \omega^{2}+\frac{c}{R^{4}}
$$


For the third term of the right hand side of (4.2), we have

$$
\begin{aligned}
-\left(\Delta_{f} \psi\right) \omega & =-\left[\left(\partial_{r} \psi\right) \Delta_{f} r+\left(\partial_{r}^{2} \psi\right) \cdot|\nabla r|^{2}\right] \omega \\
& \leq-\left[\partial_{r} \psi(\alpha+(n-1) K(R-1))+\partial_{r}^{2} \psi\right] \omega \\
& \leq\left[\left|\partial_{r}^{2} \psi\right|+(|\alpha|+(n-1) K(R-1))\left|\partial_{r} \psi\right|\right] \omega \\
& =\psi^{1 / 2} \omega \frac{\left|\partial_{r}^{2} \psi\right|}{\psi^{1 / 2}}+(|\alpha|+(n-1) K(R-1)) \psi^{1 / 2} \omega \frac{\left|\partial_{r} \psi\right|}{\psi^{1 / 2}} \\
& \leq \frac{\psi \omega^{2}}{10}+c\left[\left(\frac{\left|\partial_{r}^{2} \psi\right|}{\psi^{1 / 2}}\right)^{2}+\left(\frac{|\alpha| \cdot\left|\partial_{r} \psi\right|}{\psi^{1 / 2}}\right)^{2}+\left(\frac{K(R-1)\left|\partial_{r} \psi\right|}{\psi^{1 / 2}}\right)^{2}\right] \\
& \leq \frac{1}{10} \psi \omega^{2}+\frac{c}{R^{4}}+c \frac{\alpha^{2}}{R^{2}}+c K^{2},
\end{aligned}
$$

where the $f$-Laplacian comparison was used. Here, since $\operatorname{Ric}_{f} \geq-(n-1) K$ and $r\left(x_{1}, x_{0}\right) \geq 1$ in $B\left(x_{0}, R\right)$, we have the $f$-Laplacian comparison (see Theorem 3.1 in [23])

$$
\Delta_{f} r\left(x_{1}\right) \leq \alpha+(n-1) K(R-1),
$$

where $\alpha=\max _{\left\{x \mid d\left(x, x_{0}\right)=1\right\}} \Delta_{f} r(x)$. For the fourth term of the right hand side of (4.2), we have

$$
\begin{aligned}
\left|\psi_{t}\right| \omega & =\psi^{1 / 2} \omega \frac{\left|\psi_{t}\right|}{\psi^{1 / 2}} \\
& \leq \frac{1}{10}\left(\psi^{1 / 2} \omega\right)^{2}+c\left(\frac{\left|\psi_{t}\right|}{\psi^{1 / 2}}\right)^{2} \\
& \leq \frac{1}{10} \psi \omega^{2}+\frac{c}{\left(\tau-t_{0}+T\right)^{2}} .
\end{aligned}
$$

For the fifth term of the right hand side of (4.2), we have

$$
2(a+(n-1) K) \psi \omega \leq \frac{1}{10} \psi \omega^{2}+c(a+(n-1) K)^{2} .
$$

For the sixth term of the right hand side of (4.2), we have

$$
\frac{2 a g}{\mu-g} \psi \omega \leq \frac{1}{10} \psi \omega^{2}+\frac{c a^{2} g^{2}}{(\mu-g)^{2}} .
$$

Now at $\left(x_{1}, t_{1}\right)$, we substitute (4.3)-(4.8) to the right hand side of (4.2) and obtain

$$
\begin{aligned}
2 \psi(\mu-g) \omega^{2} \leq & \psi(\mu-g) \omega^{2}+c \frac{|\ln D-g|}{R^{4}}+\frac{\psi \omega^{2}}{2}+\frac{c}{\left(\tau-t_{0}+T\right)^{2}} \\
& +\frac{c}{R^{4}}+c \frac{\alpha^{2}}{R^{2}}+c K^{2}+c(a+(n-1) K)^{2}+\frac{c a^{2} g^{2}}{(\mu-g)^{2}} .
\end{aligned}
$$

Recalling that

$$
\mu-g \geq 1 \quad \text { and } \quad \frac{|\ln D-g|}{\mu-g} \leq 1
$$


then (4.9) implies

$$
\begin{aligned}
\psi \omega^{2} \leq & \frac{c}{R^{4}}+\frac{c}{\left(\tau-t_{0}+T\right)^{2}} \\
& +c \frac{\alpha^{2}}{R^{2}}+c K^{2}+c(a+(n-1) K)^{2}+\frac{c a^{2} g^{2}}{(\mu-g)^{2}}
\end{aligned}
$$

at space-time $\left(x_{1}, t_{1}\right)$. By some basic analysis, we claim that:

$$
\frac{g^{2}}{(\mu-g)^{2}} \leq \kappa^{2}, \quad \text { where } \quad \kappa:=\max \{|\ln D|, 1\}
$$

for all $g \leq \ln D$, where the constant $\mu:=1+\ln D$. To see this, notice that function $\frac{g^{2}}{(\mu-g)^{2}}$ has only one critical point $g=0$, and it is continuous on $(-\infty, \ln D]$ satisfying

$$
\lim _{g \rightarrow-\infty} \frac{g^{2}}{(\mu-g)^{2}}=1 \quad \text { and } \quad \lim _{g \rightarrow \ln D} \frac{g^{2}}{(\mu-g)^{2}}=|\ln D|^{2} .
$$

Hence (4.11) easily follows.

Using (4.11), inequality (4.10) becomes

$$
\left(\psi \omega^{2}\right)\left(x_{1}, t_{1}\right) \leq c\left(\frac{\alpha^{2}+1}{R^{2}}+\frac{1}{\left(\tau-t_{0}+T\right)^{2}}+K^{2}+(a+(n-1) K)^{2}+a^{2} \kappa^{2}\right),
$$

where we used $R \geq 2$. This implies that

$$
\begin{aligned}
\left(\psi^{2} \omega^{2}\right)\left(x_{1}, t_{1}\right) & \leq\left(\psi \omega^{2}\right)\left(x_{1}, t_{1}\right) \\
& \leq c\left(\frac{\alpha^{2}+1}{R^{2}}+\frac{1}{\left(\tau-t_{0}+T\right)^{2}}+K^{2}+(a+(n-1) K)^{2}+a^{2} \kappa^{2}\right) .
\end{aligned}
$$

Since $\psi(x, \tau)=1$ when $d\left(x, x_{0}\right)<R / 2$ by the proposition (2) of Lemma 2.3. from the above estimate, we have

$$
\begin{aligned}
\omega(x, \tau) & =(\psi \omega)(x, \tau) \\
& \leq(\psi \omega)\left(x_{1}, t_{1}\right) \\
& \leq c\left(\frac{|\alpha|+1}{R}+\frac{1}{\tau-t_{0}+T}+K+a+(n-1) K+a \kappa\right)
\end{aligned}
$$

for all $x \in M$ such that $d\left(x, x_{0}\right)<R / 2$. By the definition of $w(x, \tau)$ and the fact that $\tau \in\left(t_{0}-T, t_{0}\right]$ was chosen arbitrarily, we in fact show that

$$
\frac{|\nabla g|}{\mu-g}(x, t) \leq c\left(\sqrt{\frac{1+|\alpha|}{R}}+\frac{1}{\sqrt{t-t_{0}+T}}+\sqrt{K}+\sqrt{a(\kappa+1)}\right)
$$

for all $(x, t) \in Q_{R / 2, T} \equiv B\left(x_{0}, R / 2\right) \times\left[t_{0}-T, t_{0}\right]$ with $t \neq t_{0}-T$. Since $g=\ln u$ and $\mu=1+\ln D$, the theorem follows when $x_{1} \notin B\left(x_{0}, 1\right)$.

Now we consider the other case: $x_{1} \in B\left(x_{0}, 1\right)$. In this case, $\psi$ is constant in space direction in $B\left(x_{0}, R / 2\right)$ by our assumption, where $R \geq 2$. So at 
$\left(x_{1}, t_{1}\right),(4.2)$ yields

$$
\begin{aligned}
2(\mu-g) \omega & \leq \frac{\psi_{t}}{\psi}+2(a+(n-1) K)+\frac{2 a g}{\mu-g} \\
& \leq \frac{C}{\tau-\left(t_{0}-T\right)}+2(a+(n-1) K)+2 a \kappa,
\end{aligned}
$$

where we used proposition (3) of Lemma 2.3. Since $\mu-g \geq 1$ and $\psi(x, \tau)=$ 1 when $d\left(x, x_{0}\right)<R / 2$ by the proposition (2) of Lemma 2.3, the above estimate indeed gives

$$
\begin{aligned}
\omega(x, \tau) & =(\psi \omega)(x, \tau) \\
& \leq(\psi \omega)\left(x_{1}, t_{1}\right) \\
& \leq \omega\left(x_{1}, t_{1}\right) \\
& \leq \frac{C}{\tau-\left(t_{0}-T\right)}+(a+(n-1) K)+a \kappa
\end{aligned}
$$

for all $x \in M$ such that $d\left(x, x_{0}\right)<R / 2$. By the definition of $w(x, \tau)$ and the fact that $\tau \in\left(t_{0}-T, t_{0}\right]$ was chosen arbitrarily, we in fact prove that

$$
\frac{|\nabla g|}{\mu-g}(x, t) \leq \frac{C}{\sqrt{t-t_{0}+T}}+\sqrt{(n-1) K}+\sqrt{a(\kappa+1)}
$$

for all $(x, t) \in Q_{R / 2, T}$ with $t \neq t_{0}-T$. So (1.6) is still true.

\section{REFERENCES}

1. M. Bailesteanua, X.-D. Cao, A. Pulemotov, Gradient estimates for the heat equation under the Ricci flow, J. Funct. Anal. 258 (2010), 3517-3542.

2. D. Bakry, M. Emery, Diffusion hypercontractivitives, in: Séminaire de Probabilités XIX, Lecture Notes in Math., vol. 1123, Springer-Verlag, Berlin, 1985, pp. 177-206.

3. K. Brighton, A Liouville-type theorem for smooth metric measure spaces, J. Geom. Anal. 23 (2013), 562-570.

4. E. Calabi, An extension of E. Hopf's maximum principle with an application to Riemannian geometry, Duke Math. J. 25 (1957), 45-56.

5. H.-D. Cao, Recent progress on Ricci solitons, Recent advances in geometric analysis, Adv. Lect. Math. (ALM) 11, 1-38, International Press, Somerville, MA 2010.

6. X.-D. Cao, B. Fayyazuddin Ljungberg, B.-W. Liu, Differential Harnack estimates for a nonlinear heat equation, J. Funct. Anal., 265 (2013), 2312-2330.

7. S.-Y. Cheng, S.-T. Yau, Differential equations on Riemannian manifolds and their geometric applications, Commun. Pure Appl. Math. 28 (1975), 333-354.

8. F. Chung, S.-T. Yau, Logarithmic Harnack inequalities, Math. Res. Lett. 3 (1996), 793-812.

9. L. Gross, Logarithmic Sobolev inequalities, Amer. J. Math. 97 (1975), 1061-1083.

10. R. Hamilton, A matrix Harnack estimate for the heat equation, Comm. Anal. Geom. 1 (1993), 113-126.

11. R. Hamilton, The formation of singularities in the Ricci flow, Surveys in Differential Geom. 2 (1995), 7-136, International Press.

12. X.-R. Jiang, Hamilton's gradient estimates for a nonlinear parabolic equation on Riemannian manifolds, Proc. AMS, to appear.

13. B. Kotschwar, Hamilton's gradient estimate for the kernel on complete manifolds, Proc. AMS, 135 (2007), 3013-3019. 
14. J.-Y. Li, Gradient estimates and Harnack inequalities for nonlinear parabolic and nonlinear elliptic equations on Riemannian manifolds, J. Funct. Anal. 100 (1991), 233-256.

15. P. Li, S.-T. Yau, On the parabolic kernel of the Schrodinger operator, Acta Math. 156 (1986), 153-201.

16. X.-D. Li, Liouville theorems for symmetric diffusion operators on complete Riemannian manifolds, J. Math. Pure. Appl. 84 (2005), 1295-1361.

17. X.-D. Li, Hamilton's Harnack inequality and the W-entropy formula on complete Riemannian manifolds, arXiv:1303.1242.

18. Y. Li, X.-R. Zhu, Harnack estimates for a heat-type equation under the Ricci flow, J. Differential Equations, 260 (2016), 3270-3301.

19. J. Lott, Some geometric properties of the Bakry-Émery-Ricci tensor, Comment. Math. Helv. 78 (2003), 865-883.

20. L. Ma, Gradient estimates for a simple elliptic equation on complete non-compact Riemannian manifolds, J. Funct. Anal. 241 (2006), 374-382.

21. O. Munteanu, J. Wang, Smooth metric measure spaces with nonnegative curvature, Comm. Anal. Geom. 19 (2011), 451-486.

22. P. Souplet, Q S. Zhang, Sharp gradient estimate and Yau's Liouville theorem for the heat equation on noncompact manifolds, Bull. London Math. Soc. 38 (2006), 10451053.

23. G.-F. Wei, W. Wylie, Comparison geometry for the Bakry-Émery Ricci tensor, J. Diff. Geom. 83 (2009), 377-405.

24. J.-Y. Wu, Gradient estimates for a nonlinear diffusion equation on complete manifolds, J. Partial Differential Equations, 23 (2010), 68-79.

25. J.-Y. Wu, $L^{p}$-Liouville theorems on complete smooth metric measure spaces, Bull. Sci. Math. 138 (2014), 510-539.

26. J.-Y. Wu, Elliptic gradient estimates for a weighted heat equation and applications, Math. Z., 280 (2015), 451-468.

27. J.-Y. Wu, P. Wu, Heat kernels on smooth metric measure spaces with nonnegative curvature, Math. Ann., 362 (2015), 717-742.

28. J.-Y. Wu, P. Wu, Heat kernel on smooth metric measure spaces and applications, to appear in Math. Ann., DOI: 10.1007/s00208-015-1289-6

29. Y.-Y. Yang, Gradient estimates for a nonlinear parabolic equation on Riemannian manifolds, Proc. Amer. Math. Soc. 136 (2008), 4095-4102.

30. S.-T. Yau, Harmonic functions on complete Riemannian manifolds, Commun. Pure Appl. Math. 28 (1975), 201-228.

Department of Mathematics, Shanghai Maritime University, 1550 Haigang Avenue, Shanghai 201306, P. R. China

E-mail address: jywu81@yahoo.com 